\title{
The Utility of Neuromotor Retraining to Augment Manual Therapy and Vestibular Rehabilitation in a Patient with Post-Concussion Syndrome: A Case Report
}

\author{
Andrew Teare-Ketter, PT, DPT ${ }^{1}$, Alyssa LaForme Fiss, PT, PhD ${ }^{2}$, Jeffrey Ebert, PT, DPT ${ }^{3}$ \\ 1 Department of Physical Therapy, Mercer University, Atlanta, GA, USA; Division of Sports Medicine, Department of Physical Therapy and \\ Rehabilitation, Floyd Medical Center, Rome, GA, USA, ${ }^{2}$ School of Physical Therapy, Texas Women's University, ${ }^{3}$ Department of Physical Therapy, \\ Mercer University, Atlanta, GA, USA \\ Keywords: vestibular rehabilitation, post-concussion syndrome, movement system, concussion, cervicogenic \\ https://doi.org/10.26603/001c.18823
}

\section{International Journal of Sports Physical Therapy}

Vol. 16, Issue 1, 2021

\section{Background and Purpose}

Less than half of patients discharged from the emergency department post-concussion receive patient education or follow-up care, and $10-20 \%$ of individuals will develop symptoms that last longer than six months. Current research on interventions for post-concussion syndrome (PCS) shows inconsistent results, and recommendations for effective physical therapy treatment for patients with chronic PCS are lacking. The purpose of this case report is to highlight a successful, multi-system approach to physical therapy examination and treatment of a patient with chronic PCS.

\section{Case Description}

This case describes a 21-year-old male who sustained a concussion 356 days prior to evaluation. He received no follow-up treatment and reported periods of worsening symptoms since the injury. Impairments in cervical range of motion and accessory mobility, vestibular and vestibulo-ocular function, and postural stability were identified. Both cognitive and emotional symptoms were also present. The patient attended eight, sixty-minute sessions over a five-week period in an outpatient setting. Comprehensive physical therapy interventions included manual therapy, vestibular rehabilitation, and neuromotor retraining aimed at restoring proper sensory integration and midline postural orientation.

\section{Outcomes}

Outcomes included cervical spine goniometric measurements and accessory mobility assessments. Objective measures of postural stability included the Modified Clinical Test of Sensory Interaction in Balance (m-CTSIB) and the Fukuda Step Test. The Rivermead Post-Concussion Symptoms Questionnaire (RPQ) was used to subjectively assess symptom severity. At discharge, goniometric measurements returned to within normal limits except left cervical rotation active range of motion (ROM), and both the m-CTSIB and Fukuda Step Test were within normal ranges. The RPQ score reduced from 20 to 2 demonstrating symptom resolution in all items but "headache."

\section{Discussion}

PCS can affect multiple systems, necessitating a comprehensive approach to examination and intervention. Manual therapy was used to restore cervical spine ROM, vestibular rehabilitation was utilized to improve gaze stability and visual motion sensitivity, and neuromotor retraining was implemented to improve postural stability and sensory

\footnotetext{
a Corresponding Author:
}

Andrew Teare-Ketter, PT, DPT

Division of Sports Medicine, Department of Physical Therapy and Rehabilitation, Floyd Medical Center, Rome, Georgia, USA

304 Shorter Ave \#101, Rome, GA, 30165

Email: ateare-ketter@floyd.org 
integration. Physical therapists have the ability to treat multiple systems impacted with PCS, with the potential to reduce the longevity and severity of impairments for patients.

\section{Level of Evidence}

Level V

\section{BACKGROUND}

Concussions, or mild traumatic brain injuries (TBI), have become an important topic in healthcare. Research has emphasized the need for understanding the etiology of concussions and the long-term effects concussions can have both cognitively and physically. ${ }^{1}$ The notion of a disorder associated with post-concussive symptoms was once controversial, but recent research and the increasing prevalence in professional athletes, ${ }^{1}$ collegiate and high school athletes, ${ }^{1,2}$ and the general population has led to the acceptance of a Post-Concussion Syndrome (PCS). ${ }^{1}$ Concussions and PCS are not exclusive to athletes as all individuals are susceptible to mild brain injuries and the lasting impairments that can be associated with such injuries.

In the United States, there are over two million TBI-related emergency department visits each year, and mild TBIs account for $80-90 \%$ of all TBIs. ${ }^{3}$ There are between 1.7 and 3 million sport-related concussions annually, ${ }^{4}$ and $20-50 \%$ of individuals with a mild TBI will have symptoms that last longer than three months. ${ }^{5}$ Approximately, $22 \%$ of individuals with mild TBIs are functionally impaired after one year ${ }^{3}$ however, any degree of brain trauma can create lasting cognitive, emotional, and/or physical detriments that can impair an individual's functional capabilities. ${ }^{1} \mathrm{Al}$ though protocols have been enacted with athletic evaluations, less than half of all individuals who report to an emergency department after a mild TBI receive any form of education on symptomology and medical management, or are instructed to visit a medical professional for a follow-up appointment within three months. ${ }^{3}$ Furthermore, a study by Vargo et al. ${ }^{6}$ has shown that referrals to physical therapy post-concussion are not yet common; only $28 \%$ of patients were referred to physical therapy from a concussion clinic within an academic medical center, although many patients may benefit from rehabilitation during recovery. This lack of follow-up care may increase the risk of prolonged symptoms after the initial injury. ${ }^{3}$

Risk factors for prolonged recovery may include loss of consciousness at time of injury, dizziness at time of injury, amnesia surrounding time of injury, migraine symptoms acutely after initial injury, severity of acute symptoms, delayed symptom onset, history of previous concussions, or a history of psychological considerations. ${ }^{7}$ In $70-90 \%$ of concussions, symptoms resolve within two weeks, and the majority of physiologic symptoms resolve within seven to ten days. ${ }^{8}$ The standardized definitions of PCS by the International Classification of Diseases (ICD-10) as well as the Diagnostics and Statistics Manual (DSM-IV) demonstrate poor specificity and include symptoms often demonstrated with other neurologic disorders such as migraine and depression. ${ }^{7}$ To meet the criteria for PCS as defined by the DSM-IV, the patient must demonstrate symptoms longer than three months. ${ }^{7}$ These shortcomings related to diagnosis can further delay the identification of PCS.
The ICD-10 defines PCS as the presence of three or more of the following symptoms within the first month post-injury: headache, dizziness, fatigue, irritability, insomnia, and concentration or memory difficulty. ${ }^{7}$ The DSM-IV defines PCS as cognitive deficits in attention or memory and at least three or more symptoms including: headache, dizziness, fatigue, irritability, apathy, personality change, or sleep or affective disturbance. ${ }^{7}$ Symptoms of concussions can vary in presentation, intensity, and duration depending on the systems disrupted by the initial injury. Symptoms can have a neurologic, vestibular, or musculoskeletal origin or concurrent dysfunction among these systems. ${ }^{9}$

Neurologically, there is a disruption of cerebral blood flow, ionic homeostasis, and axonal health within the cerebrum at the time of injury, all of which lead to a decreaseof-consciousness and amnesia. Persistent disruption in neuronal depolarization and metabolism and in cerebrovascular function can manifest as headaches at rest, light and sound sensitivity, irritability, and fatigue. There can also be autonomic disruption that can manifest as abnormal vital sign responses such as hypotension and/or elevated heart rate at rest and with exertion, and low symptom threshold with physical activity. ${ }^{7}$

Authors have suggested that dizziness and disequilibrium experienced in patients with a TBI can be caused by vestibular pathology $40-60 \%$ of the time. ${ }^{10}$ Decreased gaze stability and postural stability experienced with an acute concussion also can be caused by vestibular involvement. ${ }^{10}$ Central vestibular dysfunction predominates the majority of concussions; however, peripheral vestibular dysfunction can also be present. ${ }^{11}$ The most common peripheral vestibular pathology post-concussion is Benign Paroxysmal Positional Vertigo (BPPV). ${ }^{10}$ A labyrinthine concussion, which is a term used to describe peripheral vestibular dysfunction following head trauma, can also be present ${ }^{10,11}$ Damage to the labyrinthine system can progress to unilateral vestibular hypofunction (UVH) and eventually to chronic unilateral vestibular hypofunction, if not compensated or addressed. Chronic UVH can produce many of the symptoms of concussions and PCS such as dizziness, disequilibrium, postural instability, motion sensitivity, diplopia, nausea and vomiting, and headaches associated with complex visuospatial enviormnets. ${ }^{7,12}$

There is also evidence describing the role of the cervical spine in post-concussion symptoms and PCS. Forces similar to those sustained with whiplash-associated injuries are often concurrent with mild TBIs and are frequently overlooked. ${ }^{8}$ Damage to the zygapophyseal and intervertebral joints and to the soft tissues of the region, particularly the upper cervical spine, can disrupt the large role the cervical spine has in proprioceptive input to the brain. ${ }^{13}$ This dysfunction can create cervicogenic headaches and muscular tension, impair postural stability and spatial orientation, and alter oculomotor control. ${ }^{13}$ Authors have suggested that symptoms of PCS persisting beyond three months are 
often of musculoskeletal origin. ${ }^{8}$ These findings stress the importance of identifying and treating impairments associated with the cervical spine and highlight the region's influence on symptomology. Additionally, authors suggest a positive correlation exists between emotional and physical symptoms, ${ }^{14}$ indicating that persistent emotional symptoms, commonly associated with neurological dysfunction, can be increased by persistent vestibular and musculoskeletal dysfunction. ${ }^{8}$

A systematic review by Brolinson ${ }^{15}$ on sport-related concussion management concluded that many of the studied treatment strategies are inconsistent and do not yet have enough scientific support for adoption as a standard treatment protocol. ${ }^{15}$ The most promising evidence demonstrates that individualized physical therapy consisting of manual therapy and sensorimotor retraining can aid in improving return to sport outcomes in patients with PCS when compared to graded aerobic exercise and rest. ${ }^{15}$ However, the literature lacks studies that demonstrate successful interventions in patients with multiple system involvement, or the utility of physical therapy as a primary intervention. Therefore, the purpose of this case report is to highlight a successful, multi-system approach to physical therapy examination and treatment of a patient with chronic PCS.

\section{CASE DESCRIPTION: SUBJECT HISTORY AND SYSTEMS REVIEW}

The subject described in this case report is a 21-year-old male who suffered a concussion 356 days prior to the physical therapy evaluation. The injury occurred during a fall out of a moving golf cart onto his left shoulder/neck region. The subject reported loss of consciousness and post-traumatic amnesia following the injury. The subject was taken to a local emergency department and CT scan results appeared negative for cerebral bleeding but identified a left temporal bone fracture not requiring medical intervention. The subject received no education on symptomology or symptom duration, and no referral or follow-up was scheduled at the time of discharge. The subject reported a history of four previous concussions, three of which were sport-related, but otherwise had no co-morbidities or significant past medical history. Only minor residual symptoms from his previous concussions were reported that all gradually resolved. The subject had constant, persistent symptoms since the injury that impaired his abilities in school as well as his social life and had restricted athletic or recreational activities, reducing his overall quality of life. The subject visited his primary physician a few weeks prior to evaluation; his cervical spine was cleared via radiographs, and he was referred to a metropolitan outpatient physical therapy clinic.

The subject was given the Rivermead Post-Concussion Questionnaire (RPQ) to assess symptomology. The RPQ is a subjective, self-report measure that encompasses 16 items, each of which is scored from 0-4 in increasing severity, assessing separate cognitive, emotional, and somatic physical factors. ${ }^{16}$ The RPQ demonstrates high test-retest and interrater reliability for both total score and individual items via spearman rank correlation coefficients $(r=0.91, r=0.87$ re- spectively). ${ }^{16}$ The subject's chief reported symptoms during the initial evaluation were neck pain and stiffness, bilateral radicular symptoms that were worse in the left shoulder and upper extremity, lightheadedness, nausea, dizziness, blurred vision and diplopia, sensitivity to light, impaired balance, slurred speech, trouble sleeping, fatigue, slower thinking, and frustration. The subject's primary complaint was his headache symptoms, which the subject reported were brought on by reading, scanning, driving, or cervical movements. The subject also reported a period of gradual worsening during the weeks and months following his injury. The subject's goals for physical therapy were to reduce the severity of symptoms experienced since the injury, primarily concerning his headache, fatigue, and neck pain symptomology, as he reported these symptoms have impacted his abilities as a college student and decreased his participation in his social life.

The subject described in this case report provided informed consent for the study and was informed that the data collected would be used for publication. This study has been approved by the Mercer University Institutional Review Board and Office of Research Compliance. The primary author providing patient care and clinical decision making was a student physical therapist at the time of subject interaction and was supervised by a licensed physical therapist.

\section{CLINICAL IMPRESSION \# 1}

The subject's mechanism of injury, with loss of consciousness and amnesia, but lack of intracranial bleeding, suggests that the subject sustained a mild traumatic brain injury or concussion. This along with other neurologic, vestibular, and musculoskeletal symptoms, and his past medical history of multiple concussions, indicated a high probability for PCS. The mechanism of injury of a fall onto the neck/shoulder region increases the likelihood of trauma to the cervical spine and/or peripheral vestibular system. The subject's headache symptoms likely indicate a vestibular or cervicogenic origin as his symptoms worsened with periods of reading, scanning, or cervical movements rather than with exercise or at rest. It was hypothesized that the subject would have impairments in cervical spine accessory and soft tissue mobility, range of motion (ROM), vestibular function including vestibular-ocular reflex and gaze stability, balance and somatosensory input, and posture. Due to the chronicity of the subject's condition and multi-system involvement, physical therapy examination and intervention targeting multiple systems was warranted.

\section{EXAMINATION}

A cardiovascular systems review indicated normal vital signs. Assessment of the subject's static posture (Figure 1) demonstrated a right lateral side bend of his cervical spine of 7 degrees, with an associated shift of the trunk to the left. As a result, there was significant asymmetry of cervical and thoracic soft tissue. Bilateral upper trapezius and sternocleidomastoid musculature were hypertrophied, with the left upper trapezius, left sternocleidomastoid, and left levator scapulae being more prominent. His right shoulder 
Table 1: Cervical goniometric range of motion assessment at both initial evaluation and discharge, reported in degrees. Measurement reflects both active and passive range of motion as identical measurements where obtained.

\begin{tabular}{|c|c|c|c|}
\hline Motion & Normal & Initial Evaluation & Discharge \\
\hline Flexion & 45 & 56 & 70 \\
\hline Extension & 45 & 40 & 52 \\
\hline Right Rotation & 70 & 44 & 73 \\
\hline Left Rotation & 70 & 56 & 63 \\
\hline Right Sidebend & 45 & 39 & 55 \\
\hline Left Sidebend & 45 & 40 & 52 \\
\hline
\end{tabular}

Table 2: Joint accessory mobility assessments at initial evaluation and discharge.

\begin{tabular}{|c|c|c|c|c|}
\hline \multirow[b]{2}{*}{ Location } & \multicolumn{2}{|c|}{ Initial Evaluation } & \multicolumn{2}{|c|}{ Discharge } \\
\hline & CPA & UPA & CPA & UPA \\
\hline $\mathrm{C} 1-\mathrm{C} 2$ & Early capsular end feel & Early capsular end feel, pain $(\mathrm{L}>\mathrm{R})$ & Normal & Normal \\
\hline $\mathrm{C} 2-\mathrm{C} 3$ & Early capsular end feel, pain & Early capsular end feel, pain $(L>R)$ & Normal & Normal \\
\hline $\mathrm{C} 3-\mathrm{C} 4$ & Early capsular end feel, pain & Early capsular end feel, pain $(\mathrm{L}>\mathrm{R})$ & Normal & Normal \\
\hline $\mathrm{C} 4-\mathrm{C} 5$ & Early capsular end feel, pain & Early capsular end feel, pain ( $L>R)$ & Normal & Normal \\
\hline $\mathrm{C} 5-\mathrm{C} 6$ & Early capsular end feel, pain & Early capsular end feel, pain $(\mathrm{L}>\mathrm{R})$ & Pain at end range & Normal \\
\hline $\mathrm{C} 6-\mathrm{C} 7$ & Early capsular end feel, pain & Early capsular end feel, pain ( $L>R)$ & Normal & Normal \\
\hline
\end{tabular}

CPA: Central posterior-anterior mobilization; UPA: Unilateral posterior-anterior mobilization

demonstrated abnormal elevation, and his right upper extremity and scapula had increased relative abduction that was also present with functional movements. He demonstrated forward head posture and left scapular protraction with slight trunk rotation to the right.

Upon palpation, tenderness, hypertrophy, and myofascial trigger points were discovered throughout his cervical and thoracic musculature with greater dysfunction on the left than the right. Both active and passive cervical ROM were assessed, and identical measurements were obtained. (Table 1). The subject reported pain and tightness with all cervical active and passive motions, with an increase in headache pain with repeated cervical movements into extension. All ROM measurements, except flexion, were below normal. ${ }^{17}$ Repeated cervical motions recreated numbness and tingling in his left upper extremity extending down to the second and third digits. as well as decreased sensation. Spurling's tests and cervical compression test were negative. Bilateral shoulder AROM tested within normal limits.

Unilateral posterior-anterior mobilizations (UPAs) of the cervical spine revealed decreased available range of movement with an early capsular end feel throughout all levels, with more restriction assessed in segments C1-C3. Increased restriction and pain was identified on the left compared to the right. Central posterior-anterior mobilizations (CPAs) of the cervical intervertebral joints also revealed decreased available range of movement with an early capsular end feel and pain throughout all levels. (Table 2). Assessment of central posterior-anterior mobilizations of the up-

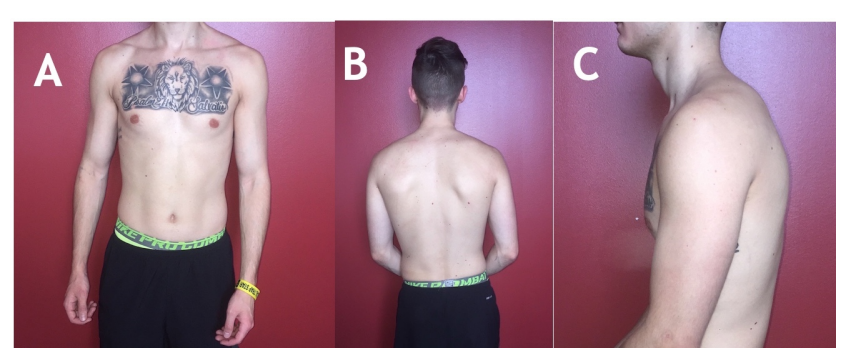

Figure 1

A: Initial view, anterior postural assessment.

B: Initial view, posterior postural assessment.

C: Initial view, lateral postural assessment.

per and middle thoracic intervertebral joints in prone identified decreased available range as well. Increased available range and pain were assessed at T6, with hypermobility identified. This segment also demonstrated increased motion with thoracic active extension. Reduced available range and pain with first rib depression also assessed on the left.

The subject's dizziness was not position-dependent and could last several minutes to hours in duration. Due to these subjective reports and time since onset, BPPV was not suspected; rather, UVH was hypothesized and a positional assessment was deferred. Tests of vestibulo-ocular reflex (VOR) gain reproduced the subject's symptoms of nausea, dizziness, and disequilibrium, and no nystagmus or saccadic intrusions were observed with ocular movements. A 
Table 3: Outcome measures at initial evaluation and discharge. Outcome measures include the Fukuda Step Test, Modified Clinical Test of Sensory Interaction in Balance, and the Rivermead Post Concussion Questionnaire.

\begin{tabular}{|c|c|c|}
\hline Outcome Measure & Initial Evaluation & Discharge \\
\hline Fukuda Step Test* & $70^{\circ}$ Rotation to Left & $20^{\circ}$ to Left \\
\hline M-CTSIB & $\begin{array}{l}\text { EO: } 3 \\
\text { EC: } 2 \\
\text { EOF: } 3 \\
\text { ECF: } 2\end{array}$ & $\begin{array}{l}\text { EO: } 3 \\
\text { EC: } 3 \\
\text { EOF: } 3 \\
\text { ECF: } 3\end{array}$ \\
\hline RPQ† & $20 / 64$ & $2 / 64$ \\
\hline \multicolumn{3}{|c|}{$\begin{array}{l}\quad{ }^{*}<45^{\circ} \text { normal result for Fukuda Step Test } \\
\dagger \geq 16 \text { on RPQ indicative of post-concussion syndrome }\end{array}$} \\
\hline
\end{tabular}

EO: Eyes open; EC: Eyes closed; EOF: Eyes open firm surface; ECF: Eyes closed firm surface;

M-CTSIB: Modified Clinical Test of Sensory Integration in Balance; RPQ: Rivermead Post-Concussion Questionnaire

formal vestibular ocular motor screening (VOMS) or test of Dynamic Visual Acuity (DVA) were not performed which are considered limitations of this report. A VOMS incorporates testing of smooth pursuit, saccades, convergence, VOR, and visual motion sensitivity and has demonstrated internal consistency and sensitivity in identifying concussions. ${ }^{18}$ DVA would have aided in quantifying and measuring impairment in gaze stability and should be included in the evaluation of similar patients.

The subject's postural stability and sensory organization were assessed using the Fukuda Step Test and the Modified Clinical Test of Sensory Integration in Balance (m-CTSIB). The Fukuda Step Test has not been shown to identify the side of a potential vestibular lesion, and the test-retest reliability should be interpreted with caution; therefore, more than one balance assessment was selected. However, this test is commonly used to screen for postural instability and vestibular dysfunction, as it is easily accessible and reproducible within a clinical setting. ${ }^{19,20}$ The subject demonstrated a 70-degree turn to the left; an abnormal response is a turn in either direction greater than 45 degrees. ${ }^{19}$

The $\mathrm{m}$-CTSIB is a shorted version of the original six-item CTSIB and is utilized to assess balance deficits and sensory organization. The original CTSIB has demonstrated good test-retest reliability and has demonstrated moderate correlation with the Sensory Organization Test (SOT) to determine sensory-related postural instability. ${ }^{21}$ The m-CTSIB is a 30-second test of independent standing scored on an ordinal scale based time and sway for four progressively difficult balance conditions: EO (eyes open, firm surface), EC (eyes closed, firm surface), EOF (eyes open, foam surface), ECF (eyes closed, foam surface). The scale ranges from 0-3 ( $0=$ unable, $1=$ fall $<30 \mathrm{~s}, 2=$ unstable, 3 =stable for $30 \mathrm{~s}) .{ }^{21}$ The differing sensory conditions investigate postural stability with varying visual and/or somatosensory input. The subject was able to maintain balance with EO for 30 seconds, but could not maintain postural stability with EC and ECF. This indicates the subject relied more heavily on somatosensory and visual feedback to maintain postural stability. The results are listed in Table 3 .

Upon item analysis of the Rivermead Post-Concussion Questionnaire, the subject's condition could be categorized as encompassing both vestibulo-ocular and cervicogenic in- volvement. A recent study determined a cutoff score with patients experiencing prolonged symptoms (at least six months post-mild TBI) that demonstrated high diagnostic accuracy of PCS as 16 or above. ${ }^{22}$ The subject scored a 20 during the initial evaluation, indicating a strong likelihood of PCS as the source of his symptoms.

\section{CLINICAL IMPRESSION \#2}

The subject examination revealed impairments that coincide with vestibular and musculoskeletal driven PCS. Limitations in cervical ROM, accessory mobility, and soft tissue extensibility were discovered. Asymmetric soft tissue hypertrophy and a myofascial trigger points on the subject's left indicated potential soft tissue dysfunction. It is possible this originated from decreased somatosensory input from the cervical spine, from vestibular dysfunction, or both. The results of the subject's vestibular exam indicated more peripheral vestibular involvement as impaired VOR gain was assessed and symptomatic, but no nystagmus or saccadic intrusions were identified with ocular movements. The subject's cervical sidebend at rest could have developed from these impairments or as a result of the initial injury, as either a compensatory offload of an injury to the cervical spine or an asymmetrical neural discharge from the peripheral vestibular system. It is hypothesized that these impairments led to further postural and movement system abnormalities. The subject's headache symptoms were likely a result of a combination of dysfunctional vestibulo-ocular reflex and cervical muscle tension. The subject's self-reported tolerance to exercise, lack of headaches at rest, and normal vitals reduced the likelihood of extensive autonomic involvement.

The multi-system involvement supported the need for a comprehensive approach to physical therapy intervention including manual therapy to aid in musculoskeletal tissue health, vestibular rehabilitation, and neuromotor retraining to improve sensory organization and balance and to reinforce optimal system integration. Decreases in pain, headache severity, fatigue, and other subjectively reported symptoms during the subject's activities of daily living were expected throughout the plan of care. It was also hypothesized that as the subject's physical symptoms subsided, 
Table 4: Interventions by session including manual therapy, soft tissue mobility, therapeutic exercise, vestibular rehabilitation, and neuromotor retraining.

\begin{tabular}{|c|c|c|c|}
\hline Session & Manual Therapy* & Soft Tissue Stretches† & $\begin{array}{l}\text { Therapeutic Exercise } \ddagger \\
\text { Vestibular Rehabilitation§ } \\
\text { Neuromotor Retraining }\end{array}$ \\
\hline $\begin{array}{l}\text { Initial } \\
\text { Evaluation }\end{array}$ & $\begin{array}{l}\mathrm{STM}^{\#} \text {, cervical and thoracic spine } \\
\text { CPAs, cervical spine UPAs, manual } \\
\text { traction, median nerve glides }\end{array}$ & $\begin{array}{l}\text { Passive: UT, LS, SCM, } \\
\text { scalenes, suboccipitals, } \\
\text { pectoralis minor }\end{array}$ & $\begin{array}{l}\text { Scapular retraction, deep cervical } \\
\text { flexion }\end{array}$ \\
\hline 2 & $\begin{array}{l}\text { Same as session one; added first } \\
\text { rib depression mobilizations }\end{array}$ & $\begin{array}{l}\text { Passive: UT, LS, SCM, } \\
\text { scalenes, suboccipitals } \\
\text { Self: pectoralis minor, } \\
\text { paraspinals }\end{array}$ & $\begin{array}{l}\text { Scapular retraction, deep cervical } \\
\text { flexion, cable rows, seated balance on } \\
\text { swiss ball, VORx1, saccades }\end{array}$ \\
\hline 3 & Same as session 2 & Same as session 2 & Same as session 2 \\
\hline 4 & Same as session 2 & $\begin{array}{l}\text { Self: UT, LS, SCM, scalenes, } \\
\text { suboccipitals, pectoralis } \\
\text { minor, paraspinals }\end{array}$ & $\begin{array}{l}\text { Scapular retraction, deep cervical } \\
\text { flexion, cable rows, cable pull downs, } \\
\text { VORx } 1 \text { and saccades } w / \text { laser Harness }\end{array}$ \\
\hline $5-8$ & $\begin{array}{l}\text { STM, cervical and thoracic CPAs, } \\
\text { cervical UPAs, manual traction, } \\
\text { first rib mobilizations }\end{array}$ & Same as session 4 & Same as session 4 \\
\hline $\begin{array}{l}\text { Home } \\
\text { Exercise } \\
\text { Program }\end{array}$ & $\begin{array}{l}\text { Self-UPAs with towel throughout } \\
\text { cervical spine, } 3 \times 30 \text { in sitting }\end{array}$ & Same as session 4 & VORx 1 to onset of symptoms, $5 x /$ day \\
\hline \multicolumn{4}{|c|}{$\begin{array}{l}\text { *Mobilizations progressed from grades I-II to grades III-IV, performed on all hypomobile cervical and thoracic segments for three sets } \\
\text { of } 30 \text { repetitions; neural glides performed in three sets of } 10 \text { or to reduction of symptoms after mobilizations } \\
\text { †Soft tissue stretches performed in two sets with } 30 \text { second hold } \\
\text { †Therapeutic exercises performed for three sets of } 10,15 \text {, or } 20 \text { repetitions depending on rate of fatigue } \\
\text { §Vestibular exercises included VORx } 1 \text { and saccades with head movements and performed in sets of three to onset of dizziness or } \\
\text { headache } \\
{ }^{* *} \text { Neuromotor retraining included performance of VORx } 1 \text { and saccades with head movements while wearing laser harness, in sitting, } \\
\text { for three sets of one minute or until onset of dizziness or headache; harness placed above ears and around trunk for sagittal plane } \\
\text { alignment, mirror placed in front for self-correction } \\
\text { \#Soft tissue mobilization to cervical and thoracic musculature included multidirectional petrissage or trigger point release to bilateral } \\
\text { UT, LS, SCM, suboccipitals, and longus colli depending on pain and tissue extensibility }\end{array}$} \\
\hline
\end{tabular}

STM: Soft tissue mobilization; CPA: Central posterior-anterior mobilization; UPA: Unilateral posterior anterior mobilization; UT: Upper trapezius; LS: Levator scapulae; SCM: Sternocleidomastoid; VOR: Vestibulo-ocular reflex

emotional symptomology would decrease as well due to the positive correlation between the two symptom subgroups. Objective improvements in cervical spine ROM and accessory mobility, a decrease in lightheadedness experienced with gaze stabilization exercises, and improvements in balance assessments were expected with intervention.

\section{INTERVENTION}

The subject attended an initial evaluation followed by seven 60-minute treatment visits over a five-week period in an outpatient physical therapy clinic. Sessions are outlined in Table 4 . The primary approach to treatment was restoring cervical spine ROM and vestibulo-ocular input, all while restoring optimal posture and sensory integration via neuromotor retraining. It was known at the time of the initial evaluation that the subject needed to be discharged from therapy after five weeks in order to return to school fulltime. The subject also reported that he would be unable to attend therapy during the second week of the plan of care, prioritizing the need for early reduction of inflammation and irritability, as well as offloading of neural structures, during the initial treatment session. The subject was pre- scribed a daily home exercise program that included selfstretching of cervical musculature and self-mobilization of the cervical spine. Additionally, gaze stabilization exercises, to be performed multiple times per day, were prescribed.

Each treatment session began with manual therapy targeting asymmetries in cervicothoracic joints and musculature. Techniques included manual traction, central and unilateral posterior-anterior cervical and thoracic joint mobilizations, soft tissue mobilizations, and active and passive stretches with emphasis placed on the hypertrophied regions. Low-velocity mobilizations were performed at all levels and gradually increased by grade and from mid to end range to reduce pain and increase overall available range of movement. Mobilizations were performed in sets of 30 repetitions. Neural glides in the median nerve pattern were also performed with alternating elbow and wrist flexion/extension in sets of 10 until reduction in neural symptoms. Soft tissue stretches were held for 30 seconds for two repetitions.

For therapeutic exercise, the subject was educated on and placed in proper posture in midline orientation with tactile cues. All exercises were performed in front of a mir- 
ror for increased positional awareness and to aid in restoring the afferent ability of the cervical and thoracic spine. Several exercises incorporated a two-sided cable system for further neuromotor input to aid in symmetrical movement patterns and postural stability. Each exercise was performed in sets of 10,15 , or 20 repetitions on the subject's rate of fatigue, in order to improve postural endurance but promote proper orientation. VOR exercises, saccadic exercises in conjunction with head movements, and habituation exercises were performed as it has been shown that vestibular substitution, adaptation, and habituation significantly reduce symptomology and improve function in patients with uncompensated UVH. ${ }^{12}$

Beginning with session 4 and performed for the remainder of the POC, the subject participated in neuromotor retraining exercises while wearing a laser system (Figure 2) to integrate improved vestibular and somatosensory function. The four-laser harness system symmetrically placed lasers above each ear and around the thorax at the level of the $12^{\text {th }}$ ribs. The system gave the subject feedback regarding sagittal plane alignment for both the trunk and head, with a mirror placed in front of the subject for self-correction. By actively aligning the lasers horizontally, the subject was able to re-associate afferent input from cervical and thoracic regions and vestibular system with symmetrical alignment, emphasizing system interaction in optimal positioning.

The subject performed VORx1, with eyes on fixed target and head rotating side to side, for vestibular adaptation of the vestibular-ocular reflex. The subject would then perform saccadic exercises in conjunction with head movements for substitution and compensation of the central nervous system for the disruption in VOR gain. The subject would do this by moving eyes from one target to another with head fixed then moving head to follow with eyes fixed. This also encouraged utilization of improved cervical ROM while restoring the cervical spine's role on in the ocular and somatosensory systems. All exercises were performed for at least three sets and to onset of dizziness to aid in habituation of visual motion sensitivity, but not performed past onset of dizziness to aid in pacing and prevention of prolonged symptom exacerbation; however, the APTA CPG recommends at least 20 minutes a day of gaze stabilization exercises for chronic UVH. ${ }^{12}$

Each session concluded with 15 minutes of heat and interferential current (IFC) electrical stimulation applied predominantly on the hypomobile and hypertrophied side of the cervical spine for pain reduction.

\section{OUTCOMES}

At the beginning of each session, the subject was reassessed subjectively regarding symptom resolution and objectively via cervical AROM, joint mobility of all cervical levels, median nerve tension, and VOR sensitivity. There was a steady improvement in his condition throughout the plan of care. By session 4, the subject's radicular symptoms and neck pain had resolved. His lightheadedness, dizziness, visual disturbances, and headaches were reported to have decreased in frequency but were dependent on prolonged activity. At the time of discharge, all of the subject's symptoms had completely resolved except for his headaches,

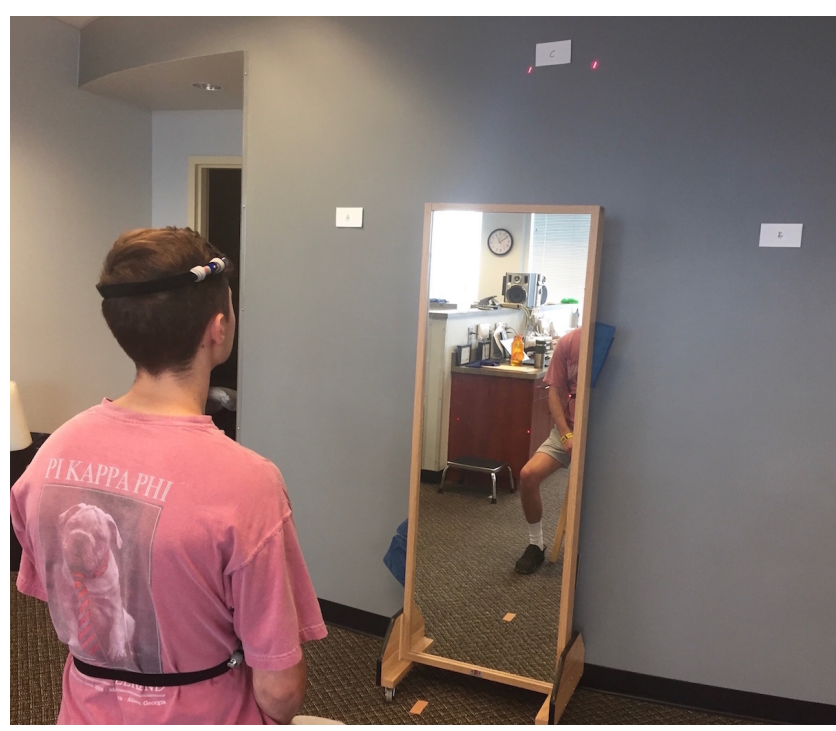

Figure 2: Performance of neuromotor training using laser harness.

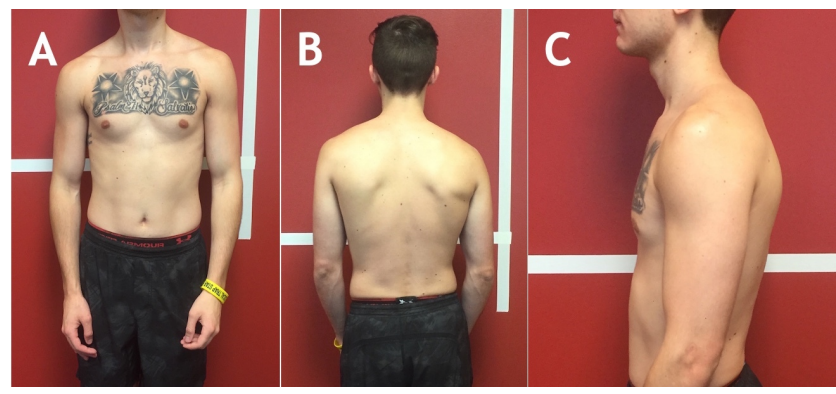

\section{Figure 3}

A: At discharge, anterior postural assessment.

B: At discharge, posterior postural assessment.

C: At discharge, lateral postural assessment.

which were reported as "mild" and "infrequent." The subject had also discontinued use of his prescription glasses, as he reported no continued diplopia.

The subject demonstrated postural improvements in all planes (Figure 3). His cervical musculature appeared to have more symmetrical definition and his forward head posture was reduced. His resting cervical lateral flexion had decreased from 7 degrees to 2 degrees and there was a significant reduction in hypertrophy of the paraspinals throughout the cervical and thoracic spine. The subject demonstrated improved scapular position and improved scapular upward rotation with overhead movements. The upper extremities also rested in a more natural position. Despite these notable postural improvements, he continued to demonstrate some postural asymmetries at discharge, most notably slight left shoulder elevation and right pelvic elevation. Ideally, his postural progression would have continued during a longer plan of care; however, the subject's symptom reduction was the primary goal and was achieved after eight sessions.

The subject's cervical ROM increased in each plane and returned to normal ranges except for left rotation, and all 
motions were no longer painful at end-range with no onset of symptoms with repeated motions (Table 1). The subject's cervical spine accessory mobility normalized bilaterally, with tenderness to palpation only experienced with CPAs at C5-C6 (Table 2). Left first rib mobility also normalized with no tenderness assessed. Increased overall available motion remained with CPAs at T6 but no pain reproduction. Cervical and thoracic myofascial restriction had reduced bilaterally with no tenderness to palpation upon assessment.

The subject's symptoms of dizziness, nausea, or diplopia could not be recreated with VOR exercises during treatment or at home, and both the Fukuda Step Test and m-CTSIB tested within normal ranges (Table 3 ). The subject displayed improved balance and postural stability with no recurrence of dizziness, lightheadedness, diplopia, or nausea while performing the Fukuda Step Test or the m-CTSIB. All but one item on the RPQ were reported as resolved, with "headaches" categorized as "mild." The subject chose to continue his home exercise program upon returning to school without physical therapy after discharge.

\section{DISCUSSION}

This case report describes the comprehensive physical therapy management of a patient with PCS that had not received any medical follow-up prior to referral for initial evaluation. The subject was almost 12 -months post-injury had since experienced symptoms including neck pain, upper extremity radicular symptoms, lightheadedness, dizziness, nausea, diplopia, light sensitivity, impaired balance, slurred speech, trouble thinking, fatigue, and slower thinking.

It was hypothesized that the majority of the subject's symptoms were the result of damage sustained to the labyrinthine vestibular system and cervical spine that, with lack of medical follow-up, were never identified or addressed. Chronic unilateral hypofunction produces many of the symptoms experienced by the subject such as nausea, dizziness, decreased postural stability and spatial orientation, motion sensitivity, diplopia, and headaches produced by complex visuospatial environments. ${ }^{12}$ Upon reflection, it is likely that partially compensated central vestibular dysfunction could also have been present considering the pathophysiology of concussion. Impairments experienced by this subject including nausea and dizziness, impaired postural stability, disrupted VOR, and motion and visual motion sensitivity could have been a result of both central and peripheral pathology. Moving forward, it is important to recognize that treatment for patients with dizziness following head trauma must be planned knowing that the central and peripheral vestibular systems, and the cervical spine can be involved. ${ }^{11}$

Impairments in cervical accessory and soft tissue mobility have been shown to create muscular tension and cervicogenic headaches, as well as decreased somatosensory and positional input to the brain leading to decreased spatial orientation and postural stability. ${ }^{13}$ It is suspected that the abnormalities in joint mobility, muscular tension, proprioceptive input, and spatial orientation created the postural deviations and impaired movement patterns displayed by the subject, which had become chronic, and likely drove further dysfunction.

The primary focus of intervention was to restore cervical spine ROM and improve vestibular function. Manual therapy and stretches aided in restoring symmetrical accessory mobility, ROM, and muscular tension. This reduced the subject's pain, normalized impairments, and reduced the hypermobility assessed in the thoracic spine. Therapeutic exercises strengthened dysfunctional postural musculature and incorporated neuromotor retraining to aid in restoring proper afferent input and functional movement patterns. The success of these interventions reinforces the benefit of individualized spinal manual therapy and sensorimotor input in treating post-concussion syndrome recognized by Brolinson; ${ }^{15}$ however, the subject in this case report presented with disruption in both the vestibular and musculoskeletal systems.

Vestibular rehabilitation included adaptation and substitution of the vestibular-ocular reflex, as well as habituation exercises for motion and visual motion sensitivity. VORx1 targeted adaptation and recovery of disrupted VOR gain. Horizontal and vertical saccades in conjunction with head movements was performed to allow an intact central vestibular function to substitute for disrupted VOR gain and increase gaze stability. These vestibulo-ocular exercises and cervical movements were performed to onset of dizziness to aid in the habituation of motion and visual motion sensitivity. As decreased gaze stability and motion and visual sensitivity can be a result of both central and peripheral vestibular pathology, the interventions would have been appropriate for central dysfunction although a peripheral abnormality was hypothesized.

The laser harness system gave the subject increased postural awareness in an attempt to increase somatosensory and proprioceptive input. The visual feedback provided by the lasers improved the vestibular system's ability to detect, regulate, and maintain position sense. This reduced the subject's disequilibrium while also restoring the proprioceptive role of the cervical spine. This intervention was selected primarily because the subject displayed both vestibular and musculoskeletal involvement. The exercise allowed rehabilitation of the two systems simultaneously and emphasized neuromotor retraining as the subject was also able to self-correct abnormal movement patterns and regulate proper posture during functional movements.

The reduction in vestibulo-ocular related symptoms and in headaches reported supports the findings stated by the APTA's clinical practice guideline on unilateral vestibular hypofunction that vestibular adaptation, substitution, and habituation are effective. ${ }^{12}$ As the subject's vestibular and musculoskeletal symptoms reduced throughout the plan of care, so too did the subject's reported symptoms of trouble sleeping, fatigue, frustration, difficulty concentrating, and slowed thinking, echoing data suggesting that emotional and cognitive symptoms can also be driven by prolonged physical symptoms. ${ }^{14}$

The results of the interventions above demonstrate the importance of a comprehensive approach to evaluation and treatment of a patient with chronic post-concussion syndrome, as well as the efficacy of physical therapy as a referral destination. As discussed, PCS can present with multiple etiologies that can become muddled with chronicity; how- 
ever, the subject was successfully evaluated and treated. A plan of care was established with comprehensive interventions targeting multiple systems that, except for infrequent and mild headaches, led to complete resolution of the subject's symptoms. The subject was almost 12-months postinjury and received no follow-up care after discharge from an ER, highlighting the importance of medical follow-up after concussion. The success of this case also demonstrates that physical therapy can serve as a routine referral destination after more significant brain injury has been ruled out.

Limitations to this study include lack of more sensitive and objective vestibulo-ocular testing, including the VOMS or DVA, to quantify central and peripheral dysfunction and gaze stability. Subjective symptom-specific outcome measures such as the Headache Disability Index, Dizziness Handicap Inventory, or Visual Vestibular Analog Scale could also have been utilized. The use of a graded treadmill test upon initial examination would have aided in assessing the role, or lack thereof, of autonomic involvement in the subject's condition. Due to the multi-system involvement of PCS, it is difficult to identify an intervention that was most beneficial in this case, which can be considered a limitation; however, the purpose of this case report is to demonstrate a comprehensive, multi-system approach to evaluation and treatment of PCS. Further research is warranted on the use of neuromotor retraining in conjunction with comprehensive interventions in athletes and other types of patients with PCS. Research should also investigate patient outcomes of physical therapy as the primary referral post-injury.

\section{CONCLUSION}

The results described in this case report detail a successful comprehensive approach to treating a patient with chronic post-concussion syndrome who reported multiple symptoms and several risk factors for prolonged dysfunction. The subject's condition demonstrated involvement of multiple systems which necessitated a comprehensive examination and plan of care. Using neuromotor retraining along with manual therapy and vestibular rehabilitation targeted both the musculoskeletal and vestibular systems and aided in restoration of proper function of both systems simultaneously, impacting the overall movement system. Improving posture and correcting movement incoordination as a result of prolonged dysfunction was an important component. This episode of care was beneficial for this subject, even though the subject was almost a year removed from injury, and suggests that physical therapy may be impactful in prolonged recovery from post-concussion syndrome.

\section{CONFLICTS OF INTEREST}

All authors disclose no conflicts of interest.

Submitted: December 29, 2019 CDT, Accepted: July 24, 2020 CDT 


\section{REFERENCES}

1. American Association of Neurological Surgeons. Concussion. 2018. https://www.aans.org/Patients/Ne urosurgical-Conditions-and-Treatments/Concussion; Accessed December 4, 2018.

2. Giza CC, Prins ML, Hovda DA. It's not all fun and games: Sports, concussions, and neuroscience. Neuron. 2017;94(6):1051-1055. doi:10.1016/i.neuro n.2017.05.003

3. Seabury SA, Gaudette E, Goldman DP, et al. Assessment of follow-up care after emergency department presentation for mild traumatic brain injury and concussion. JAMA. 2018;1(1).

4. University of Pittsburgh Medical Center. Concussion facts and statistics. 2019. https://www.up mc.com/services/sports-medicine/services/concussio n/facts-statistics; Accessed December 14, 2018.

5. Faux S, Sheedy J, Delaney R, Riopelle R. Emergency department prediction of post-concussive syndrome following mild traumatic brain injury-an international cross-validation study. Brain Inj. 2011;25(1):14-22. doi:10.3109/02699052.2010.531686

6. Vargo MM, Vargo KG, Gunzler D, Fox KW. Interdisciplinary rehabilitation referrals in a concussion clinic cohort: An exploratory analysis. $P M \& R$. 2016;8(3):241-248. doi:10.1016/j.pmrj.2015.0 $\underline{7.006}$

7. Ellis MJ, Leddy J, Willer B. Multi-disciplinary management of athletes with post-concussion syndrome: An evolving pathophysiological approach. Front Neurol. 2016;7:136. doi:10.3389/fneur.2016.001 $\underline{36}$

8. Morin M, Langevin P, Fait P. Cervical spine involvement in mild traumatic brain injury: A review. J Sport Med. 2016;2016:1-20. doi:10.1155/2016/15901 $\underline{61}$

9. Quatman-Yates CC, Hunter-Giordano A, Shimamura KK, et al. Physical therapy evaluation and treatment after concussion/mild traumatic brain injury. J Orthop Sports Phys Ther. 2020;50(4):CPG1-CPG73.

10. Kolev OI, Sergeeva M. Vestibular disorders following different types of head and neck trauma. Funct Neurol. 2016;31(2):75-80. doi:10.11138/fneur/2 $\underline{016.31 .2 .075}$

11. Furman JM, Whitney SL. Central causes of dizziness. Phys Ther. 2000;80(2):179-187. doi:10.109 3/ptj/80.2.179
12. Hall CD, Herdman SJ, Whitney SL, et al. Vestibular rehabilitation for peripheral vestibular hypofunction: An evidence-based clinical practice guideline. $J$ Neurol Phys Ther. 2016;40(2):124-155. doi:10.1097/np t.0000000000000120

13. Kristjansson E, Ther M, Treleaven J. Sensorimotor function and dizziness in neck pain: Implications for assessment and management. J Orthop Sports Phys Ther. 2009;39(5):364-377. doi:10.2519/jospt.2009.283 $\underline{4}$

14. Potter S, Leigh E, Wade D, Fleminger S. The Rivermead Post Concussion Symptoms Questionnaire: A confirmatory factor analysis. J Neurol. 2006;253(12):1603-1614. doi:10.1007/s0041 5-006-0275-Z

15. Brolinson PG. Management of sport-related concussion: A review. Clin J Sport Med. 2014;24(1):89-90. doi:10.1097/jsm.000000000000006 2

16. King NS, Crawford S, Wenden FJ, Moss NEG, Wade DT. The Rivermead Post Concussion Symptoms Questionnaire: A measure of symptoms commonly experienced after head injury and its reliability. $J$ Neurol. 1995;242(9):587-592. doi:10.1007/bf00868811

17. Reese NB, Bandy WD. Normative range of motion of the extremities and spine in adults. In: Joint Range of Motion and Muscle Length Testing. 3rd ed. St. Louis, MO: Elsevier; 2017.

18. Mucha A, Collins MW, Elbin ER, et al. A brief Vestibular/Ocular Motor Screening (VOMS) assessment to evaluate concussions: Preliminary findings. Am J Sports Med. 2014;42(10):2479-2486. do $\mathrm{i}: 10.1177 / 0363546514543775$

19. Zhang YB, Wang WQ. Reliability of the Fukuda Stepping Test to determine the side of vestibular dysfunction. J Int Med Res. 2011;39(4):1432-1437.

20. Bonanni M, Newton RA. Test-retest reliability of the Fukuda Stepping Test. Physiother Res Int. 1998;3(1):58-68. doi:10.1002/pri.122

21. Freeman L, Gera G, Horak FB, Blackinton MT, Besch M, King L. The Instrumented Test of Sensory Integration for Balance: A validation study. J Geriatr Phys Ther. 2018;41(2):77-84. doi:10.1519/jpt.0000000 000000110 
22. Thompson C, Davies P, Herrmann L, Potter S,

Summer M. Approaches to establishing validated cut-

off scores on the Rivermead Post Concussion

Symptoms Questionnaire (RPQ). Brain Inj.

2016;30(5-6):770. 